\title{
Diffuse Large B-Cell Lymphoma of the Lacrimal Sac in a Japanese Patient
}

\author{
Satoshi Kakutani Yasuhiro Takahashi Ma. Regina Paula Valencia \\ Hirohiko Kakizaki \\ Department of Oculoplastic, Orbital, and Lacrimal Surgery, Aichi Medical University \\ Hospital, Nagakute, Japan
}

\section{Keywords}

Lacrimal sac · Diffuse large B-cell lymphoma - Japanese patients

\begin{abstract}
Purpose: To report a Japanese patient with diffuse large B-cell lymphoma (DLBCL) in the lacrimal sac. Methods: This is a case report of a 52-year-old Japanese woman who presented with a 3-month history of epiphora on the right side and a 1-month history of swelling on the right medial canthal area. Lacrimal sac irrigation showed patency of the lacrimal drainage system. Imaging studies revealed a lacrimal sac mass with involvement of the nasolacrimal duct. $\boldsymbol{R e}$ sults: Histopathology and immunohistochemistry of the biopsy specimen revealed DLBCL. Systemic workup revealed no other lesion. The patient received 6 cycles of $\mathrm{R}-\mathrm{CHOP}$ regimen. After completion of the 5th cycle of R-CHOP, all of the symptoms had resolved. Conclusion: We report a case of DLBCL in the lacrimal sac, which is the most common type of lacrimal sac lymphoma in Japan. Since DLBCL is one of the aggressive types of lymphoma, Japanese patients with lacrimal sac lymphoma tend to have a poor prognosis.
\end{abstract}




\section{Introduction}

Lacrimal sac lymphoma is a relatively rare condition. Diffuse large B-cell lymphoma (DLBCL) and extranodal marginal zone lymphoma of mucosa-associated lymphoid tissue (MALT lymphoma) are the most common histological types of lacrimal sac lymphoma in the UK [1]. In Japan, we reported 5 consecutive cases of primary lacrimal sac lymphoma, most of whom presented with DLBCL [2]. Here, we report an additional case of DLBCL in the lacrimal sac.

\section{Case Report}

A 52-year-old woman presented with a 3-month history of epiphora on the right side and a 1-month history of swelling in the right medial canthal area. There was no prior history of ocular or systemic disease.

On the first examination, a mass was palpated in the subcutaneous area of the right medial canthus (Fig. 1a). Lacrimal apparatus irrigation revealed a patent lacrimal drainage system. Computed tomography of the orbits revealed a lacrimal sac mass with involvement of the nasolacrimal duct. The mass was homogenous and isodense with respect to muscle, and there was no bone erosion. Magnetic resonance imaging revealed an isointense to slightly hyperintense mass on both T1- and T2-weighted images with mild enhancement (Fig. 1b). The results of blood tests were unremarkable.

Excisional biopsy via external approach was performed under local anesthesia. The specimens showed proliferation of large-sized lymphocytes on histopathological examination (Fig. 1c). Immunohistochemical examination revealed that the specimens were positive for CD20, CD10, CD19, and BCL-6, but negative for CD3, CD5, BCL-2, and MUM-1 (Fig. 1d, e). The Ki67 labeling index was $80 \%$. The results were compatible with DLBCL, germinal center B-cell type. No extraorbital lesion was found (Ann Arbor Stage IE). The patient was referred to a hematologist and was given 6 cycles of R-CHOP regimen, with good response. After completion of the 5th cycle of R-CHOP, the epiphora and the palpable mass on the right medial canthus had resolved.

\section{Discussion}

Here, we report a case of DLBCL in the lacrimal sac. In the UK, one-third of lacrimal sac lymphomas are MALT lymphomas, while the other one-third are DLBCLs [1]. On the other hand, 13 patients with primary lacrimal sac lymphoma have been reported in Japan, including our previously reported 5 patients: 6 patients $(46.2 \%)$ had DLBCL, while only 2 patients $(15.4 \%)$ had MALT lymphoma [2, 3]. Incorporating the results of this report to the previous studies $[2,3]$ reveals that half of the reported cases (7 of 14 patients) were found to have DLBCL. Since DLBCL is a more aggressive type of lymphoma than MALT lymphoma, lacrimal sac lymphoma tends to show poorer prognosis among Japanese compared to the UK patients.

Epiphora is usually the presenting symptom of a lacrimal sac lymphoma, as the presence of the tumor in the lacrimal sac causes obstruction of the tear drainage system [4,5]. However, this case showed a patent lacrimal drainage system. Among our previously reported cases, 4 of 5 cases also presented with a patent lacrimal drainage system [2]. Therefore, despite the absence of epiphora or the patency of the lacrimal drainage system, lacrimal sac lymphomas 
should also be considered and ruled out when we are presented with a mass in the medial canthal area.

The imaging findings in our patient were typical for lacrimal sac lymphoma [2]. Computed tomography revealed a homogenous and isodense mass with respect to muscle. Magnetic resonance imaging showed an isointense to slightly hyperintense mass on both T1- and T2weighted images, with mild enhancement. Although bone destruction was not observed in this patient, this can occasionally be seen in some cases due to extensive growth of the mass [6]. Unfortunately, we were not able to take a dacryocystography, which would have been helpful to confirm the mass in the lacrimal sac and evaluate patency of the lacrimal drainage system.

In conclusion, we have reported a case of lacrimal sac DLBCL. In Japan, since half of lacrimal sac lymphomas are found to be DLBCLs, lacrimal sac lymphoma tends to show a poorer prognosis.

\section{Statement of Ethics}

The authors adhered to the tenets of the 1964 Declaration of Helsinki. Institutional review board approval and written informed consent from the patient for the publication of this report were obtained.

\section{Disclosure Statement}

The authors declare that there is no conflict of interest. This work received no funding.

\section{References}

1 Sjö LD, Ralfkiaer E, Juhl BR, Prause JU, Kivelä T, Auw-Haedrich C, et al.; European Organization for Research and Treatment of Cancer. Primary lymphoma of the lacrimal sac: an EORTC ophthalmic oncology task force study. Br J Ophthalmol. 2006 Aug;90(8):1004-9.

2 Sabundayo MS, Takahashi Y, Kakizaki H. Lacrimal sac lymphoma: a series of Japanese patients. Eur J Ophthalmol. 2018 Oct:1120672118803510 [Epub ahead of print].

3 Kajita F, Oshitari T, Yotsukura J, Asanagi K, Baba T, Kishimoto T, et al. Case of primary diffuse large B-cell lymphoma of lacrimal sac in a Japanese patient. Clin Ophthalmol. 2010 Nov;4:1351-4.

4 Montalban A, Liétin B, Louvrier C, Russier M, Kemeny JL, Mom T, et al. Malignant lacrimal sac tumors. Eur Ann Otorhinolaryngol Head Neck Dis. 2010 Nov;127(5):165-72.

5 Flanagan JC, Stokes DP. Lacrimal sac tumors. Ophthalmology. 1978 Dec;85(12):1282-7.

6 Kitaguchi Y, Takahashi Y, Mupas-Uy J, Takahashi E, Kakizaki H. Primary marginal zone B-cell lymphoma of the mucosa-associated lymphoid tissue of the lacrimal sac found with epiphora: a case report. Case Rep Ophthalmol. 2016 Sep;7(3):148-54. 


\section{Case Reports in Ophthalmology}
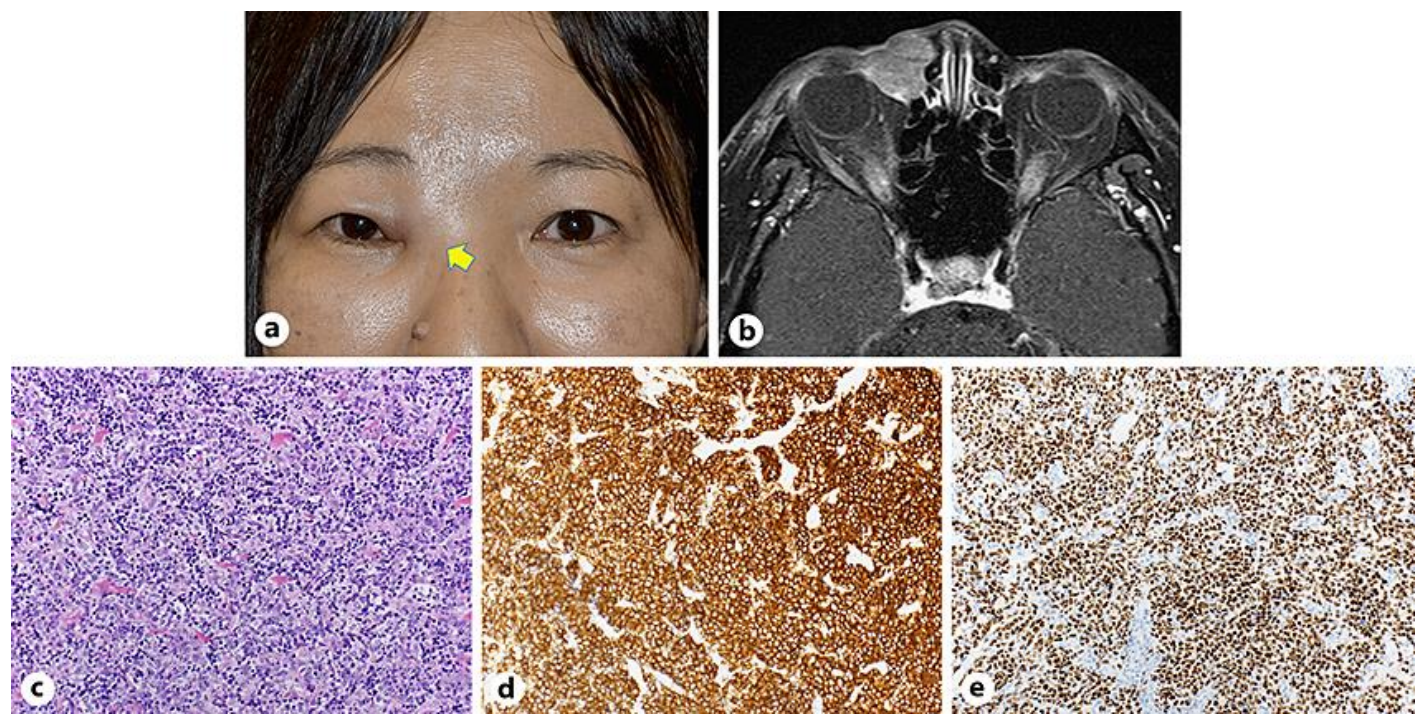

Fig. 1. a The patient's photograph during the first examination showing a mass in the right medial canthus. b Enhanced T1-weighted axial magnetic resonance imaging showed a soft tissue mass in the lacrimal sac with heterogeneously mild enhancement. c-e Histopathological and immunostaining specimens from the lacrimal sac. c Diffuse proliferation of large lymphoid cells with high-density nuclei (hematoxylin and eosin, $\times 200$ ). Immunostaining with CD20 (d) and BCL-6 (e) were both positive $(\times 200)$. 